\title{
Multiwavelength polarization observations of the $\gamma$-ray bright quasar PKS 0420-014
} \author{
P.S. Smith 6 \\ ${ }^{1}$ Astron. Inst., St.-Petersburg State Univ., Russia e-mail: vlar@astro.spbu.ru \\ ${ }^{2}$ Institute for Astrophysical Research, Boston University, MA, USA \\ ${ }^{3}$ University of Crete, Heraklion, Greece \\ ${ }^{4}$ Joint Institute for VLBI in Europe, Dwingeloo, Netherlands \\ ${ }^{5}$ Instituto de Astrofisíca de Andalucía, CSIC, Granada, Spain \\ ${ }^{6}$ Steward Observatory, University of Arizona, Tucson, AZ, USA
}

I.S. Troitskiy ${ }^{1}$, D.A. Morozova1 ${ }^{1}$, S.G. Jorstad ${ }^{1,2}$, A.P. Marscher ${ }^{2}$, V.M. Larionov ${ }^{1}$, D.A. Blinov ${ }^{3,1}$, I. Agudo ${ }^{4,5,2}$, and

\begin{abstract}
We analyze total and polarized intensity images of the quasar PKS 0420-014 obtained monthly with the VLBA at $43 \mathrm{GHz}$ during 2008-2012 along with $\gamma$-ray data provided by the Fermi Large Area Telescope and multi-color photometric and polarimetric measurements collected by different optical telescopes. During this period the quasar underwent a number of optical flares, which were accompanied by rapid rotation of polarization angle, an increase of activity in $\gamma$-rays, and the appearance of new superluminal knots in the parsec-scale jet. We investigate the fine structure of the flares at different wavelengths and in polarized light, and determine kinematic parameters of the knots. We compare the rapid evolution of the optical polarization with the polarization of the VLBI core and knots. We interpret the multi-wavelength behavior within a model that places the blazar "dissipation zone" at the millimeter-wave core of the parsec-scale jet.
\end{abstract}

\section{Introduction}

The blazar PKS 0420-014 ( $\mathrm{z}=0.915)$ is strongly variable at all wavelengths from $\gamma$-rays to radio waves. The quasar was among the first X-ray sources detected by ROSAT [1] and among the first $\gamma$-ray blazars identified by EGRET [2], and it is listed in the First Fermi Large Area Telescope (LAT) Catalog of $\gamma$-ray sources [3]. Britzen et al. [4] have found superluminal motion with $\beta_{a p p} \approx 2-14 \mathrm{c}$ for five jet components at $8.4 \mathrm{GHz}$ and Jorstad et al. [5] have measured similar velocities of $7.6 \pm 3.5$ and $10.9 \pm 2.8$ at 43 GHz. The VLBI history of 0420-014 shows that the jet has changed its direction dramatically at $43 \mathrm{GHz}: \theta \approx 280^{\circ}$ in 1998-2001 [5], $\theta \approx 180^{\circ}$ in 2005 [6], and $\theta \approx 100^{\circ}$ from 2008 to the present (BU group [9]). In addition, a weak region of emission at $43 \mathrm{GHz}$ is always visible at $\theta \sim 180^{\circ}$. At $15 \mathrm{GHz}$ the jet has $\theta \sim 200^{\circ}$ and is curved toward the east, while on kiloparsec scales the jet points directly toward the south, where two lobes are apparent (1.4 GHz, [7]).

\section{Observations and data reduction}

We obtain optical (R-band) flux densities from photometric observations at the $0.4 \mathrm{~m}$ telescope of St. Petersburg State U. (LX200) and $0.7 \mathrm{~m}$ telescope of the Crimean Astrophysical Observatory (AZT-8). The data analysis for these telescopes is described in [8]. We also use R-band data from the Perkins Telescope (BU group [9]), Liverpool Telescope, Calar Alto Telescopes [10], and Bok and Kuiper telescopes of Steward Observatory [11].

We use total and polarized intensity images of the quasar obtained at $43 \mathrm{GHz}$ with the VLBA roughly monthly. We have modelled the images in I, Q and U Stokes parameters by circular components with Gaussian brightness distribution to obtain total flux density, position with respect to the core, fractional polarization, and electric vector position angles (EVPA) of each component. The "core" is a stationary feature located at the upstream end of the portion of the jet that is visible at $43 \mathrm{GHz}$. Identification of components across the epochs is based on analysis of their distance from the core, flux density, position angle and size. We have computed the kinematic parameters of knots (proper motion, velocity, and acceleration) by fitting the positions of a component over epochs by different polynomials of order from 1 to 4 in the same manner as described in [5].

We use total flux and polarization data of UMRAO, OVRO, and the SMA at cm- and mm-wavelengths to supplement our optical light curves.

We have derived $0.1-200 \mathrm{GeV} \gamma$-ray flux densities using the data provided by the Large Area Telescope (LAT) of the Fermi Gamma-ray Space Telescope. We have used the unbinned likelihood analysis method implemented in the standard Fermi Science Tools software [12] for data reduction. The $\gamma$-ray light curve is constructed 


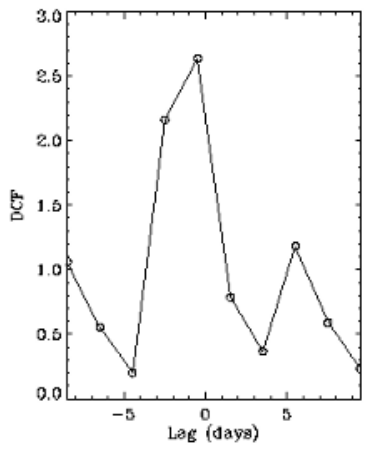

Figure 1. Discrete correlation function between $\gamma$-ray and optical R-band light curves

with a 7 day integration time and with a detection criterion that the maximum-likelihood test statistic (TS) should exceed 10.0.

\section{Results and Discussion}

\subsection{Light curves}

Since the beginning of the Fermi LAT observations the quasar underwent two periods of enhanced activity separated by a long quiescent state at $\gamma$-ray and optical wavelengths. The active periods are characterized by a number of individual flares, an increase of the degree of polarization, a fast rotation of the optical EVPA, and appearance of new superluminal knots in the parsec-scale jet. The light curves and polarization parameters vs. time curves during the period from August 2008 to February 2013 are presented in Fig. 2. The flux density peaks are very sharp at both $\gamma$-ray and optical wavelengths. During the largest flare (MJD 56151.5) the R band flux changes from $6.1 \mathrm{mJy}$ to $3.7 \mathrm{mJy}$ over a two-day interval. A cross-correlation analysis of the optical and $\gamma$-ray data does not show any significant lag between variations in these bands (Fig. 1). The radio light curves at 230, 14.5, 8.0 GHz and at $43 \mathrm{GHz}$ for the VLBI core are smoother, but in general replicate the long- term behavior at optical and $\gamma$-ray wavelengths.

In addition to the long-term correlated activity across the whole electromagnetic spectrum, a number of short synchronous flares are apparent at $\gamma$-ray and optical wavelengths. The local maxima in $\gamma$-rays are marked in Fig. $2 \mathrm{a}$ by the vertical gray solid lines. The majority, if not all, of these local peaks have counterparts in $\mathrm{R}$ band if optical data are available, although a 7-day binning of $\gamma$-ray photons limits the specification of an exact position of the peaks.

The positions of the local $\gamma$-ray and optical R-band peaks in flux are presented in Table 1 . The average lag between the peaks at the two wavelengths is $0.7 \pm 3.0$ days, which is within the size of a bin of $\gamma$-rays. Therefore, as in the case of a cross-correlation analysis there is no an apparent significant lag between the sharply defined flares in the light curves.

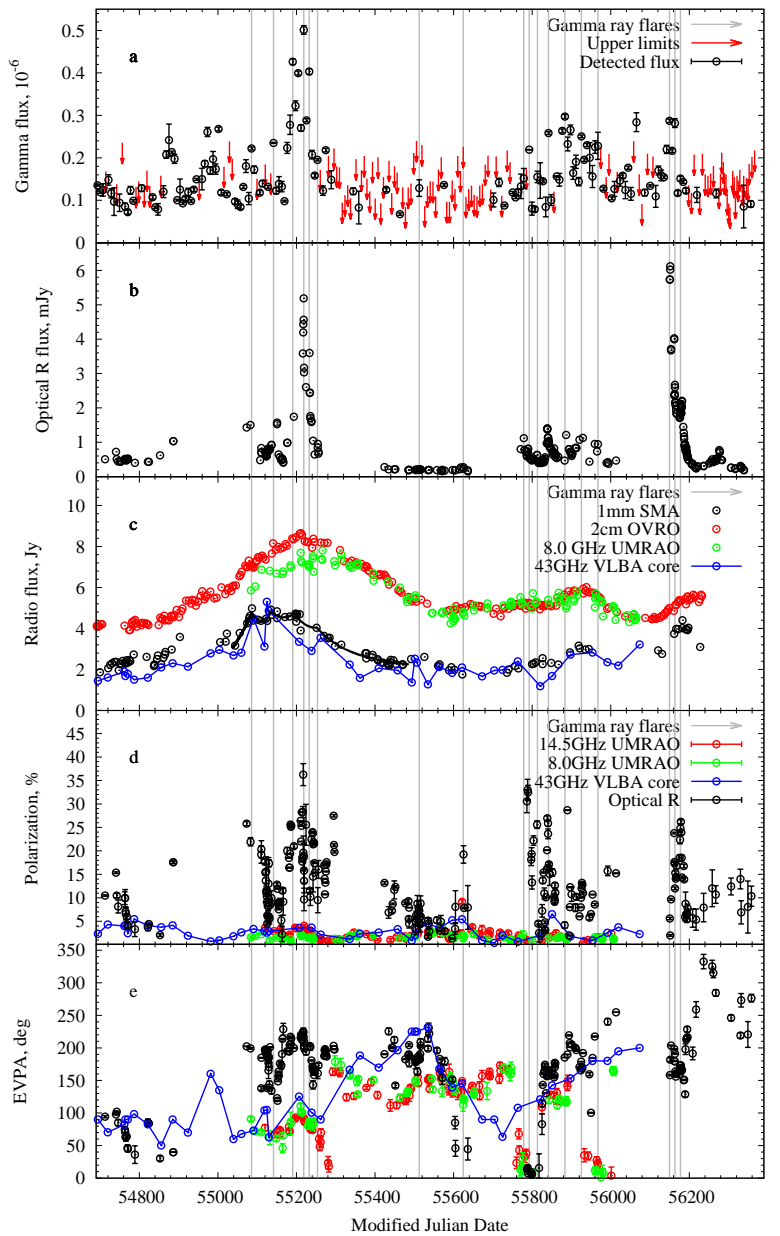

Figure 2. Light curves and polarization parameter curves. a: Gamma-ray light curve in units of $\mathrm{ph} \cdot \mathrm{cm}^{-2} \cdot \mathrm{s}^{-1}$, red arrows mark upper limits. b: Optical light curve in $\mathrm{R}$ band. c: Light curves in radio bands at 230,15 , and $8.0 \mathrm{GHz}$, and $43 \mathrm{GHz}$ for the VLBA core. The black solid line shows a model of four flares at $1 \mathrm{~mm}$ by components with exponential rise and decay. $\mathbf{d}$ : Degree of polarization at $14.5 \mathrm{GHz}$ and $8.0 \mathrm{GHz}$, in the $43 \mathrm{GHz}$ VLBA core, and optical $\mathrm{R}$ band. e: EVPAs at $14.5 \mathrm{GHz}$ and $8.0 \mathrm{GHz}$, in the $43 \mathrm{GHz}$ VLBA core, and optical $\mathrm{R}$ band.

The flux at the mm- and cm-wavelengths changes more smoothly than at higher energies. A crosscorrelation analysis between the radio and optical light curves reveals a peak which is statistically significant but wide. We have applied the method proposed by Valtaoja et al. (1999) [13] to study short timescale variability at $1 \mathrm{~mm}$. We have fitted flares in the $1 \mathrm{~mm}$ light curve by a sum of four components with an exponential rise and decay. The resulting model curve is presented in Fig. 2c by the black solid line. The time of these four peaks coincides with the local peaks of the $\gamma$-ray and optical light curves within 1-7 days. Short timescale variability at $2 \mathrm{~cm}$ is less prominent and local peaks of the light curve at $8 \mathrm{GHz}$ are not aligned with peaks of the $\gamma$-ray and optical light curves. 
Table 1. Positions of flux peaks in $\gamma$-ray, optical $\mathrm{R}$, and $1 \mathrm{~mm}$ radio bands

\begin{tabular}{|c|c|c|c|c|c|}
\hline \multicolumn{2}{|c|}{ Peak in $\gamma$-rays (MJD) } & \multicolumn{2}{c|}{ Peak in optical R band } & \multicolumn{2}{c|}{ Peak in 1mm band } \\
\hline MJD & Flux (ph cm s $^{-1}$ ) & MJD & Flux (mJy) & MJD & Flux (Jy) \\
\hline 55085.5 & $2.22 \mathrm{e}-7 \pm 3.52 \mathrm{e}-9$ & 55082.9 & $1.500 \pm 0.023$ & 55086.7 & $4.98 \pm 0.25$ \\
55141.5 & $2.35 \mathrm{e}-7 \pm 1.24 \mathrm{e}-9$ & 55150.5 & $1.571 \pm 0.007$ & 55134.6 & $4.92 \pm 0.25$ \\
55190.5 & $4.26 \mathrm{e}-7 \pm 6.58 \mathrm{e}-9$ & 55193.4 & $1.742 \pm 0.027$ & 55197.3 & $4.72 \pm 0.26$ \\
55218.5 & $5.01 \mathrm{e}-7 \pm 1.01 \mathrm{e}-8$ & 55218.3 & $5.188 \pm 0.153$ & & \\
55232.5 & $4.03 \mathrm{e}-7 \pm 6.71 \mathrm{e}-9$ & 55233.2 & $3.596 \pm 0.073$ & & \\
55253.5 & $1.95 \mathrm{e}-7 \pm 2.52 \mathrm{e}-9$ & 55254.2 & $0.954 \pm 0.042$ & 55251.2 & $4.04 \pm 0.23$ \\
55778.5 & $1.52 \mathrm{e}-7 \pm 2.32 \mathrm{e}-8$ & 55778.7 & $1.118 \pm 0.011$ & & \\
55792.5 & $2.19 \mathrm{e}-7 \pm 3.59 \mathrm{e}-10$ & 55789.6 & $0.815 \pm 0.024$ & & \\
55813.5 & $1.55 \mathrm{e}-7 \pm 1.35 \mathrm{e}-8$ & 55812.5 & $0.635 \pm 0.005$ & & \\
55841.5 & $2.58 \mathrm{e}-7 \pm 3.33 \mathrm{e}-9$ & 55838.5 & $1.402 \pm 0.009$ & & \\
55883.5 & $2.97 \mathrm{e}-7 \pm 4.75 \mathrm{e}-9$ & 55886.5 & $1.213 \pm 0.003$ & & \\
55925.5 & $2.50 \mathrm{e}-7 \pm 3.16 \mathrm{e}-9$ & 55929.3 & $1.127 \pm 0.015$ & & \\
55967.5 & $2.28 \mathrm{e}-7 \pm 3.20 \mathrm{e}-8$ & 55967.4 & $0.941 \pm 0.022$ & & \\
56149.5 & $2.87 \mathrm{e}-7 \pm 4.01 \mathrm{e}-9$ & 56151.5 & $6.123 \pm 0.011$ & & \\
56163.5 & $2.82 \mathrm{e}-7 \pm 1.04 \mathrm{e}-8$ & 56161.5 & $4.023 \pm 0.015$ & & \\
56177.5 & $1.51 \mathrm{e}-7 \pm 5.72 \mathrm{e}-9$ & 56178.6 & $2.117 \pm 0.012$ & & \\
\hline
\end{tabular}

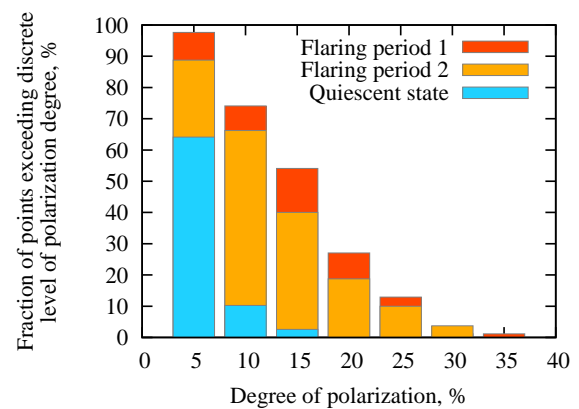

Figure 3. Percentage of points exceeding discrete levels of polarization degree in optical $\mathrm{R}$ band for the following three periods: the first flaring period on MJD 55073-55297, the quiescent state on MJD 55424-55637, and the second flaring period on MJD $55787-56196$

\subsection{Polarization}

Over the presented interval of observations the quasar PKS 0420-014 displays significant variability of the degree of optical polarization and a large span of EVPA rotation. During the highest $\gamma$-ray maximum we observed a very high degree of the optical polarization, more than $35 \%$.

The activity at optical and $\gamma$-ray wavelengths fades during the period from August 2010 to June 2011, and the degree of optical polarization differs significantly from the period of high activity. The histogram in Fig. 3 shows that only $12.5 \%$ of measurements have polarization degrees $>10 \%$ during the quiescent state, while for the first flaring period more than $70 \%$ of measurements have polarization degrees $>10 \%$, with $66 \%$ of the measurements exceeding the $10 \%$ level for the second activity period.

The degree of polarization at $8.0 \mathrm{GHz}$ and $14.5 \mathrm{GHz}$ changes from $<1 \%$ to $4-6 \%$ during the maxima. An exception is the single point at $14.5 \mathrm{GHz}$ with a polarization of $9.16 \%$ at MJD 55621.5. This point corresponds to

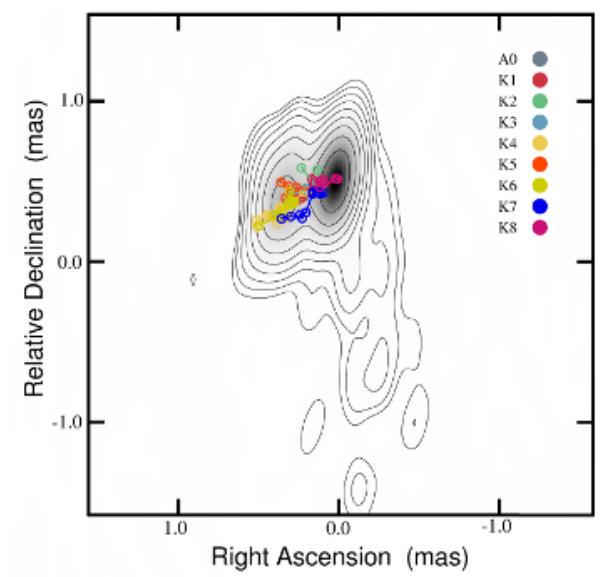

Figure 4. VLBA-image of the quasar at $43 \mathrm{GHz}$ with trajectories of the knots superposed

a rapid increase of the degree of polarization in $\mathrm{R}$ band, from $7-8 \%$ to $19.2 \%$.

In general, the EVPAs at the optical and radio wavelengths do not show a tight connection, however during the interval from MJD 55080 to 55280 the difference between the EVPAs at the optical band and VLBA core at $43 \mathrm{GHz}$ was consistently close to 90 degrees. During the time period from MJD 55534 to 55596 the EVPA of the VLBA core shows a synchronous rotation along with the optical EVPA, similar to that found by D'Arcangelo et al., 2007 [6].

\subsection{VLBA images}

The parsec-scale jet of the quasar is strongly dominated by the VLBI core, which has a complex polarization pattern (see Figs. 2d, 2e), implying multi-component substructure in the core region. We have detected 8 moving components with apparent velocities from $6.8 \mathrm{c}$ to $25.3 \mathrm{c}$. 
0420-014

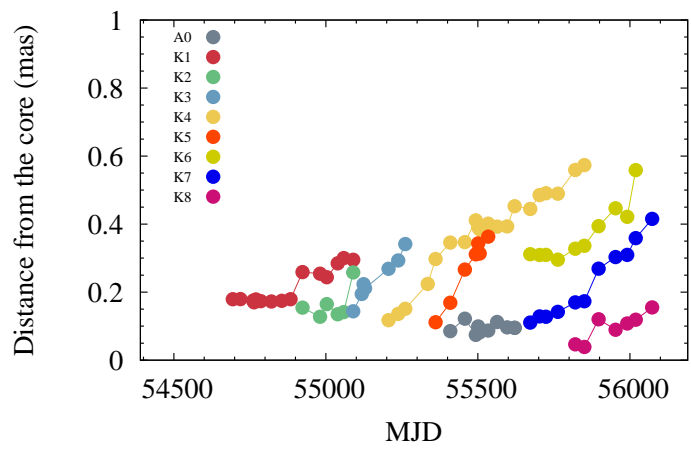

Figure 5. Positions of knots in the jet with respect to the core as a function of time

Table 2. Parameters of the knots

\begin{tabular}{|c|c|c|c|}
\hline Knot & $\beta_{a p p}(\mathrm{c})$ & $\mu(\mathrm{mas} / \mathrm{yr})$ & $T_{\text {eject }}(\mathrm{MJD})$ \\
\hline K1 & $6.75 \pm 0.18$ & $0.142 \pm 0.004$ & \\
K2 & $13.43 \pm 3.35$ & $0.282 \pm 0.070$ & \\
K3 & $16.58 \pm 1.39$ & $0.348 \pm 0.029$ & $54994 \pm 40$ \\
K4 & $13.08 \pm 1.48$ & $0.274 \pm 0.031$ & $55161 \pm 11$ \\
K5 & $25.30 \pm 0.49$ & $0.531 \pm 0.010$ & $55286 \pm 1$ \\
K6 & $11.55 \pm 0.72$ & $0.242 \pm 0.015$ & \\
K7 & $12.99 \pm 1.03$ & $0.272 \pm 0.022$ & $55564 \pm 5$ \\
K8 & $9.08 \pm 0.29$ & $0.190 \pm 0.006$ & $55702 \pm 7$ \\
\hline
\end{tabular}

Notes: $\beta_{a p p}$ - apparent velocity, c; $\mu$ - angular speed, mas/yr

The parameters of these components are presented in Table 2. The VLBA image (epoch Nov. 2010) with the trajectories of the components superposed is shown in Fig. 4. The light curve at $1 \mathrm{~mm}$ is similar to the light curve of the VLBI core at $43 \mathrm{GHz}$ which has flares during the emergence of new components.

The ejection time of knot K3 (MJD 54994) is close to the peak in $\gamma$-ray flux (MJD 55001.5). The ejection time of knot K4 (MJD 55161) corresponds to a period from 55150 to 55176 that includes a fast EVPA rotation, an increase of the degree of polarization in optical $\mathrm{R}$ band, and flares in $\gamma$-ray and optical bands. The ejection time of knot K5 (MJD 55286) corresponds to a fast rise in degree of optical polarization from 10.7\% (MJD 55273) to $27.5 \%$ (MJD 55295). The ejection time of component K7 (MJD 55564) is close to the local peak in $\gamma$-ray flux (MJD 55575). Synchronous rotation of the EVPA in the VLBA core at $43 \mathrm{GHz}$ and optical $\mathrm{R}$ band from MJD 55536 to MJD 55596 was observed as component K7 passed through the core.

\section{Summary}

During the period from August 2008 to February 2013 a number of flares occurred in PKS 0420-014 from radio to $\gamma$-ray wavelengths. We compare the light curves across the electromagnetic spectrum and find no significant lag between $\gamma$-ray and optical R-band flux variation. Short flares in the optical and $\gamma$-ray light curves coincide within
$0.7 \pm 3.0$ days, which is less than the 7-day integration interval of the $\gamma$-ray data. The times of the four peaks modelled in the $1 \mathrm{~mm}$ SMA light curve coincide with the corresponding $\gamma$-ray peaks within 1-7 days.

We have detected 8 new superluminal knots in the parsec-scale jet at $43 \mathrm{GHz}$. Apparent speeds of the knots range from $6.8 \mathrm{c}$ to $25.3 \mathrm{c}$. The epochs of ejection of knots $\mathrm{K} 3, \mathrm{~K} 4, \mathrm{~K} 5$ and $\mathrm{K} 7$ are close to the times of $\gamma$-ray and/or optical flares. They coincide with the intervals of significant rotations of EVPA and an increase in degree of optical polarization.

We infer from the behavior of the multi-wavelength flux and polarization that the $\gamma$-ray and optical events are co-spatial, and that the majority of these flares occur in the vicinity or downstream of the mm-wave VLBI core as the result of a shock-in-jet formation [14] [15].

\section{Acknowledgements}

We thank M. Aller for access to the UMRAO data. We also acknowledge The Owens Valley Radio Observatory (OVRO) for online-data access. The Submillimeter Array is a joint project between the Smithsonian Astrophysical Observatory and the Academia Sinica Institute of Astronomy and Astrophysics, and is funded by the Smithsonian Institution and the Academia Sinica. The research at St. Petersburg State University was partly funded by RFBR grants 12-02-31193 and 12-02-00452. The VLBA is an instrument of the National Radio Astronomy Observatory. The National Radio Astronomy Observatory is a facility of the National Science Foundation, operated under cooperative agreement by Associated Universities, Inc. The research at Boston U. was partly funded by NASA Fermi Guest Investigator grants NNX08AV65G, NNX11AQ03G, and NNX11AO37G. Paul Smith acknowledges funding support from NASA Fermi Guest Investigator grants NNX08AW56G and NNX09AU93G. I. Agudo acknowledges funding support from the Spanish Ministry of Economy and Competitiveness and the Regional Government of Andalucia grants AYA2010-14844 and P09-FQM-4784.

\section{References}

[1] Brinkmann, W., et al., A\&A 281, 355 (1994)

[2] Fichtel, C.E. et al., ApJS 94, 551 (1994)

[3] Abdo, A.A. et al., ApJS 188, 405A (2010)

[4] Britzen, S., et al., A\&A 360, 65B (2000)

[5] Jorstad, S.G., et al., AJ 130, 1418-1465 (2005)

[6] D'Arcangelo, F.D., et al., ApJ 659, 107-110 (2007)

[7] Cooper, , N.J., et al., ApJ Supl 171, 376 (2007)

[8] Larionov, V.M., et al., A\&A 492, 389 (2008)

[9] http://www.bu.edu/blazars/VLBAproject.html

[10] http://www.iaa.es/ iagudo/research/MAPCAT/

[11] http://james.as.arizona.edu/ psmith/Fermi

[12] Atwood, W.B., et al., ApJ 697, 1071A (2009)

[13] Valtaoja, E., et al., ApJS 120, 95 (1999)

[14] Marscher, A.P., Gear, W.K., ApJ 298, 114-127 (1985)

[15] Aller, M.F., et al. (these proceedings) 